William J. Ehlenbach

\title{
The impact of patient preferences on physician decisions in the ICU: still much to learn
}

Received: 13 May 2013

Accepted: 15 May 2013

Published online: 14 June 2013

(C) Springer-Verlag Berlin Heidelberg and ESICM 2013

This editorial refers to articles available at doi 10.1007/s00134013-2976-y and doi 10.1007/s00134-013-2977-x.

\section{W. J. Ehlenbach $(\bowtie)$}

Division of Pulmonary and Critical Care Medicine, Department of Medicine, University of Wisconsin School of Medicine and Public Health, MFCB 5245, MC 2281,

1685 Highland Avenue, Madison, WI 53705, USA

e-mail: wjehlen@medicine.wisc.edu

Tel.: +1-608-2620802

\section{W. J. Ehlenbach}

Division of Geriatrics and Gerontology, Department of Medicine, University of Wisconsin School of Medicine and Public Health, MFCB 5245, MC 2281, 1685 Highland Avenue, Madison, WI 53705, USA

Making decisions about the use of life-sustaining therapies (LST) in critically ill patients is a complicated process that requires the consideration of expected benefits and potential burdens of critical care therapies, accounts for patient goals and preferences, and confronts the reality of constrained resources [1]. The importance of this decision-making process is highlighted by the fact that the majority of deaths in European and North American ICUs involve withholding or withdrawing LST [2-6]. Decisions about the use of LST, and particularly ICU triage decisions, often lack transparency. There is evidence that patient age frequently influences triage decisions in ways that are neither based upon empirically informed estimates of expected benefit nor ethically justifiable [7, 8]. There is broad agreement that effective communication between physicians and patients is a key part of effective and just decision-making, but the context of this communication frequently adds to its complexity; physicians and patients (and their surrogate decision makers) are often meeting for the first time in the midst of an impending or ongoing health catastrophe. Advanced care planning seeks to clarify goals and preferences prior to the development of a health crisis, but the effectiveness of commonly used tools has been limited [9-12].

François Philippart, Maitè Garrouste-Orgeas, and colleagues $[13,14]$ present two studies that explore decisionmaking about LST among octogenarians and critical care physicians using hypothetical scenarios. In the first study, they explore the preferences of cognitively intact subjects aged 80 years and older regarding three life-sustaining therapies through the use of professionally produced films depicting common clinical scenarios, including actual patient footage of non-invasive ventilation (NIV), invasive mechanical ventilation (IMV), and renal replacement therapy (RRT). This novel use of videos in advanced care planning is intriguing, since it can eliminate variability in the information presented about LST and might narrow the gap between patient and physician understanding of the burdens of LST $[15,16]$. While there is no evidence of this in the present study, care must be taken to avoid using frightening imagery that risks coercing, rather than informing, patients about these therapies because the ICU environment is so foreign to many patients.

The rate of refusal of LST was strikingly high in this cohort of functionally independent subjects with chronic illness. After viewing the films, more than $1 / 4$ would refuse NIV for pulmonary edema, more than $40 \%$ of the subjects would refuse IMV for pneumonia, and nearly $2 / 3$ would refuse RRT for renal failure that developed in the ICU. One of the most common reasons given for a decision to forego LST in this cohort was a concern for the loss of independence after critical illness, echoing the fact that many older patients and patients with chronic disease value quality of life above short-term survival [17]. In effect, these patients have identified outcomes 
that could be worse than death, or at least not sufficiently better than death to justify the burdens of the therapies necessary to sustain life.

In the second study, a simulation study of physician decision-making, the physician subjects initially made decisions in regarding the use or withholding of LST based upon the characteristics of subjects from the first study, including comorbidities and functional status, as well as details of a simulated acute illness, but without direct input from patients or their families (other than what might be previously documented regarding patient preferences). This mimics the way that decisions about LST must frequently be made in the care of decompensating patients. These physicians deemed the three LSTs to be indicated and appropriate in the majority of cases, and were far more likely to use these therapies than the patients were willing to accept them. They were more likely to employ LST in younger patients, those with a higher degree of functional independence, and those without recent hospitalizations. Not surprisingly, nonpatient factors influenced decision making. Physicians practicing in Paris (where, presumably, LSTs are more commonly employed) were significantly more likely to use LST in this study, and ICU bed availability strongly influenced decisions. The extremely poor inter-physician agreement on decisions about LST in individual patients, with kappa values ranging from 0.11 for NIV to 0.24 for IMV, is a concerning finding. Hospital and physicianlevel variability in decisions about withholding or withdrawing LST, which has also been shown in other studies, is ethically problematic and raises the concern that the values (and perhaps biases) of the physician and system pressures may commonly play a role in these decisions $[18,19]$.

The most interesting aspect of this study was the way that physician knowledge of patient preferences impacted the physician subjects' decisions. Not all of the physicians changed their decision when informed of patient preferences discordant with the physician's original decision, but the majority of changes that did occur involved the physician deciding not to use a LST after learning of patient preferences not to receive it. This suggests the importance of physicians understanding patient preferences up front, even if the ideal mechanism by which such preferences should be determined and communicated remains unknown. Their findings underscore the fact that, in the absence of knowledge of patient preferences, physicians are more likely to provide LSTs to patients who would not want them rather than to refuse such therapies to patients who desire them. While instances of the latter type remain important and continue to generate considerable scholarship, it is the former situation-the use of LST that an individual patient would not have wanted-that is likely far more common and in need of improvement [20].

How results from this French study can be generalized to patients and physicians elsewhere in the world remains unknown. Culture influences perceptions of quality of life, and the balance between patient autonomy and physician paternalism varies considerably between regions and countries. Questions about the durability of patient preferences over time remain, even if technology such as videos can improve the information patients use in making those decisions. Nonetheless, this work should provide the impetus for further study of ways to improve patients' advanced care planning as well as physician decision-making about the use of LST and to allow patients' values and preferences to influence decisions made from the earliest stages of a critical illness.

Conflicts of interest The corresponding author states that there is no conflict of interest.

\section{References}

1. Task Force of the American College of Critical Care Medicine, Society of Critical Care Medicine (1999)

Guidelines for intensive care unit admission, discharge, and triage. Task

Force of the American College of Critical Care Medicine, Society of Critical Care Medicine. Crit Care Med 27(3):633-638

2. Prendergast TJ, Claessens MT, Luce JM (1998) A national survey of end-of-life care for critically ill patients. Am J Respir Crit Care Med 158(4):1163-1167
3. Esteban A et al (2001) Withdrawing and withholding life support in the intensive care unit: a Spanish prospective multi-centre observational study. Intensive Care Med 27(11):1744-1749

4. Cook DJ et al (2001) Cardiopulmonary resuscitation directives on admission to intensive-care unit: an international observational study. Lancet 358(9297): 1941-1945

5. Keenan SP et al (1998) Withdrawal and withholding of life support in the intensive care unit: a comparison of teaching and community hospitals. The Southwestern Ontario critical care research network. Crit Care Med 26(2):245-251
6. Ferrand E et al (2001) Withholding and withdrawal of life support in intensivecare units in France: a prospective survey. French LATAREA group. Lancet 357(9249):9-14

7. Sprung CL et al (2012) The eldicus prospective, observational study of triage decision making in European intensive care units. Part II: intensive care benefit for the elderly. Crit Care Med 40(1):132-138

8. Kaarlola A, Tallgren M, Pettila V (2006) Long-term survival, quality of life, and quality-adjusted life-years among critically ill elderly patients. Crit Care Med 34(8):2120-2126 
9. Teno JM et al (1997) Do advance directives provide instructions that direct care? SUPPORT investigators. Study to understand prognoses and preferences for outcomes and risks of treatment. J Am Geriatr Soc 45(4):508-512

10. Fagerlin A, Schneider CE (2004) Enough. The failure of the living will. Hastings Cent rep 34(2):30-42

11. Thompson T, Barbour R, Schwartz L (2003) Adherence to advance directives in critical care decision making: vignette study. BMJ 327(7422):1011

12. The SUPPORT Principal Investigators (1995) A controlled trial to improve care for seriously ill hospitalized patients. The study to understand prognoses and preferences for outcomes and risks of treatments (SUPPORT).

The SUPPORT principal investigators. JAMA: J Am Med Assoc 274(20):1591-1598
13. Garrouste-Orgeas M et al (2013) Simulation study of determinants and variability of ICU physician decisions in patients aged 80 or over: the ETHICA study (part II). Intensive Care Med. doi:10.1007/s00134-013-2977-x

14. Philippart F et al (2013) The ETHICA study (part I): elderly's thoughts about intensive care unit admission for lifesustaining treatments. Intensive Care Med. doi:10.1007/s00134-013-2976-y

15. Volandes AE et al (2013) Randomized controlled trial of a video decision support tool for cardiopulmonary resuscitation decision making in advanced cancer. J Clin Oncol: Off J Am Soc Clin Oncol 31(3):380-386

16. Rhondali W et al (2013) Patientphysician communication about code status preferences: a randomized controlled trial. Cancer
17. Fried TR et al (2002) Understanding the treatment preferences of seriously ill patients. N Engl J Med

346(14):1061-1066

18. Wilkinson DJ, Truog RD (2013) The luck of the draw: physician-related variability in end-of-life decisionmaking in intensive care. Intensive Care Med

19. Decato TW et al (2013) Hospital variation and temporal trends in palliative and end-of-life care in the ICU. Critical Care Med

20. Luce JM (2010) A history of resolving conflicts over end-of-life care in intensive care units in the United States. Crit Care Med 38(8):1623-1629 